\title{
Cinq leçons géographiques sur les mémoires
}

Five geography lessons about memory

Olivier Lazzarotti

\section{OpenEdition}

\section{Journals}

Édition électronique

URL : https://journals.openedition.org/insituarss/425

DOI : $10.4000 /$ insituarss.425

ISSN : 2680-4972

\section{Éditeur}

Ministère de la Culture

Référence électronique

Olivier Lazzarotti, "Cinq leçons géographiques sur les mémoires », In Situ. Au regard des sciences sociales [En ligne], 1 | 2019, mis en ligne le 15 octobre 2019, consulté le 16 décembre 2022. URL : http://journals.openedition.org/insituarss/425 ; DOI : https://doi.org/10.4000/insituarss.425

Ce document a été généré automatiquement le 16 décembre 2022.

\section{(c) (i) (9)}

Creative Commons - Attribution - Pas d'Utilisation Commerciale - Pas de Modification 4.0 International - CC BY-NC-ND 4.0

https://creativecommons.org/licenses/by-nc-nd/4.0/ 


\title{
Cinq leçons géographiques sur les mémoires
}

Five geography lessons about memory

\author{
Olivier Lazzarotti
}

1 Partons du «patrimoine ». Dans un sens élargi, autrement dit au-delà de sa portée religieuse, économique ou familiale voire génétique, etc., le mot " patrimoine » est couramment employé, plus particulièrement depuis le début des années 1960 (Lazzarotti 2012). Il peut être privé ou public, culturel ou naturel, vert ou bleu, local, national, européen ou, mieux encore, mondial, matériel ou immatériel, quand il n'est pas une ligne fiscale. Bref, à la profusion des usages répond, à l'occasion, la confusion des sens.

2 Cette confusion viendrait-elle de son emploi pour désigner une constante historique, le patrimoine traversant siècles et lieux (Choay 1991, 2009) ? Viendrait-elle de son champ sans cesse élargi au point qu'il faut multiplier les épithètes pour répondre à une diversification qui, à l'occasion, rencontre les logiques marketing d'un marché, économique voire politique, qui s'en délecte? Viendrait-elle d'un effet de concurrence entre des sciences qui se revendiquent comme (seules) légitimes pour s'attribuer le droit d'en fixer la définition? Dans le champ, Alain Bourdin (1984), réactive une sociologie déjà présente avec Maurice Halbwachs (1994) dans le milieu des années 1920. Avec Joël Candau (1996), Pierre-Henri Jeudy (2001) ou Laurier Turgeon (2003), etc., l'anthropologie participe au mouvement. Tout autant que l'histoire, d'André Chastel (1994) à Sarah Farmer (1994), Dominique Poulot (2014) ou France A. Yates (1975)... Et avec les travaux de Xavier Greffe (2003), le patrimoine s'ouvre au champ économique. Quant à la philosophie, elle n'est pas en reste, d'Henri Bergson (1990) à Paul Ricœur (2000). Et rien n'est dit des juristes. Tout cela pourrait donner l'impression qu'il tourne autour du champ comme une dispute pour la «vérité » aussi bien que pour la « légitimité » patrimoniale.

3 Vue autrement, une telle confusion ne viendrait-elle pas aussi de la popularité même du mot, multiplement entretenue, de la célébration des « Journées du patrimoine » à la fréquentation du « Patrimoine mondial »? Le patrimoine mobilise les uns et les autres, 
tous unanimes pour en faire l'éloge, quand ils n'en chantent pas les louanges. Curieuse situation, sans doute un peu paradoxale, qui en fait l'un des opérateurs des transformations des sociétés humaines, comme le moteur d'un mouvement qui, à l'occasion et par exemple, tourne comme l'alibi des processus de « gentrification »?

Affiche des Journées du patrimoine, 2001.

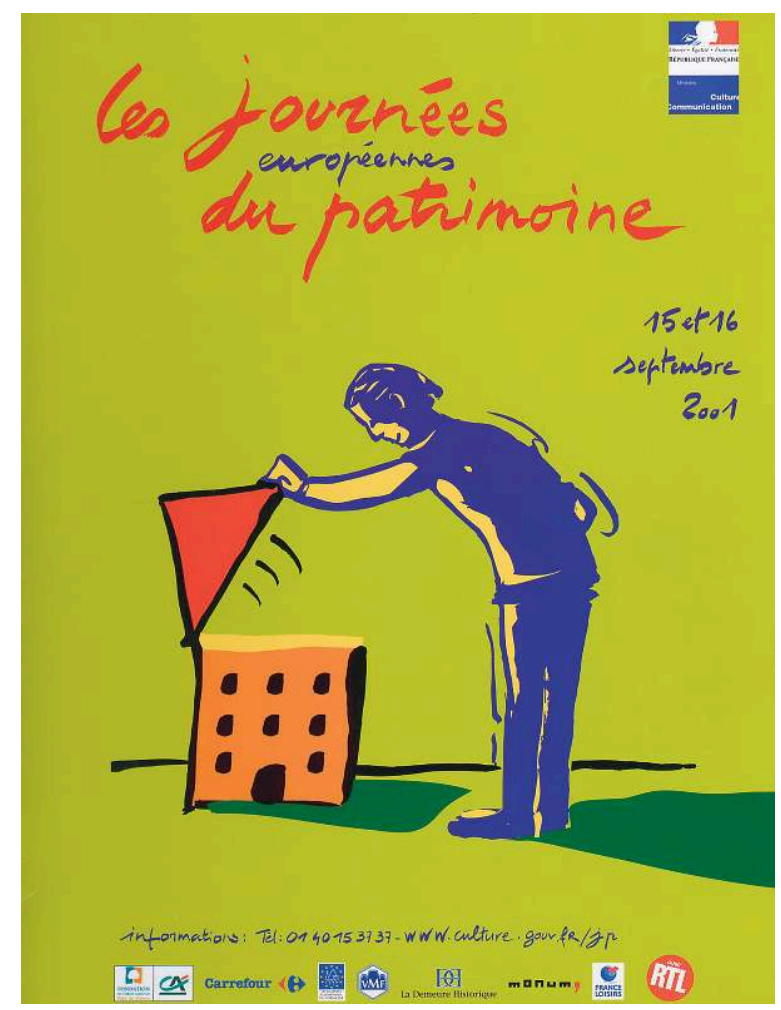

Le patrimoine, nouveau « saint » du calendrier européen ?

(c) ministère de la Culture.

La géographie participe aussi au mouvement, parfois en reprenant ses plus classiques partages. Le patrimoine est alors pris du côté de son versant « naturel» (Depraz 2008; Héritier 2009) ou bien il est analysé du point de vue de la géographie sociale (Ashworth, Graham \& Turnbridge 2000; Vincent Veschambre 2008). Plus épistémologique, une autre approche suit les relations entre géographie et patrimoine, comme c'est le cas d'Anne Hertzog (2011) et de Vincent Veschambre (2007) encore. Une réflexion, pourtant, reste un peu en suspens. Elle est celle-là même qu'invite à engager le thème de ce premier numéro de notre nouvelle revue. Car, aussi partagée par l'ensemble des sciences sociales et humaines, la thématique du patrimoine en défie les habituels mode de partage. Classiquement, ces différentes sciences se distinguent par leur sujet, par la spécificité de ce qu'elles étudient. Or, et la question patrimoniale en atteste, ces découpages tiennent de plus en plus difficilement. Cela ne veut pas dire qu'il n'y a plus de différences entre ces sciences. Mais que leurs singularités tiennent dans celles de leurs entrées dans le sujet.

5 Comment décrire cette dynamique scientifique pour la science géographique? Et comment celle-là ne conduirait-elle pas à inscrire la notion de patrimoine dans un vaste champ mémoriel, quitte à mieux y revenir pour en saisir la spécificité ? Le projet 
de cet article est donc ambitieux, trop peut-être au regard de ses exigences formelles. Ouvrir de larges perspectives reviendra aussi, de fait, à renoncer à certaines précisions.

\section{Localiser}

6 Telle une évidence fondatrice, la première des singularités de la science géographique est d'entrer dans les thématiques des sciences sociales et humaines par les voies de l'espace. De fait, le lien entre espace et mémoire est très ancien, comme le montre France A. Yates (1975) qui parle d'« art de la mémoire " pour désigner des techniques de lieux et d'images liées à l'architecture elle-même. Mais c'est dans l'Europe du tournant des XVIII ${ }^{e}$ et $\mathrm{XIX}^{\mathrm{e}}$ siècles que les lieux deviennent, de manière courante et répandue, matière à mémoires (Lazzarotti 2011). C'est que l'espace devient la page sur laquelle est gravé et montré ce qui mérite d'être retenu, tout à la fois le mémorable ou l'oubliable.

7 La nouveauté est donc européenne, mais elle a été précipitée de manière à la fois tragique et spectaculaire dans et par les tourmentes de la Révolution française. La destruction de la Bastille durant les années 1790, révèle, au-delà de celle du bâtiment lui-même, par le vide et la perte, la finitude des régimes politiques et sociaux, fussentils ceux qui auraient pu passer pour les plus inébranlables. La décapitation du roi Louis XVI, en janvier 1793, au-delà de la mort de l'homme, interrompt la chaîne familiale dynastique. Désormais, l'histoire de France ne se déroulera plus au rythme des successions générationnelles de la famille régnante. Les États-nations en construction trouveront dans leur «sol» les précieux matériaux d'une narration continue dont les termes doivent assurer la continuité de leur histoire et légitimer, par l'ancienneté, l'existence et l'unité de leurs habitants. La France se définirait-elle alors, nationalement, par son «sol», comme le suggère l'historien et homme ô combien politique François Guizot dans son rapport au roi Louis-Philippe $(1830)^{1}$ : «Les monuments historiques dont le sol de la France est couvert font l'admiration et l'envie de l'Europe savante. »Cette période est ainsi marquée par un détournement non pas de mémoire mais du processus de mémorisation, disons d'une généalogie à une géologie. La nouveauté est politique, certes, mais elle implique aussi des pans entiers de la société, comme le montrent les analyses de Joseph Morsel à propos de la notion de «traces" et de ses usages: "Tout semble donc indiquer que la notion de "traces" a commencé à être utilisable en histoire à partir de la fin du XVIII ${ }^{e}$ siècle, en Allemagne, en France ou en Angleterre, c'est-à-dire d'emblée dans le contexte de formation de la science historique » (Morsel 2016).

8 La Révolution ne fait donc pas que détruire. Tout en produisant un immense vide mémoriel, elle ouvre, dès 1790 , le débat des contenus et des modalités de production d'une mémoire renouvelée. De fait, les questions ne sont pas seulement de savoir si tel ou tel objet doit être, ou non, retenu comme mémoire nationale. En liaison avec ces questions, ce qui est en cause et débattu, est la problématique du choix des processus de mémorisation. De ce point de vue, deux conceptions s'opposent. L'une milite pour les «monuments historiques ». Elle est soutenue par l'antiquaire-naturaliste AubinLouis Millin qui propose que les érudits choisissent les bâtiments à conserver, ceux qui représenteront l'histoire du pays. L'autre conception, défendue par François Puthod de Maisonrouge, voit dans l'institution muséale un outil pédagogique et de formation citoyenne. Une volonté de transmission et l'attention portée au public inspirent ainsi ce 
qui relève de la catégorie du « patrimoine national ». Deux « régimes mémoriels » sont exposés de la sorte. Tous deux impliquent les lieux et, par eux, se présentent comme une "publicisation» des mémoires. Mais ils se distinguent par leurs processus de mémorisation qui privilégie, le premier les érudits, le second les visiteurs.

Voilà posé un principe de partition qui va faire long feu en structurant pendant près d'un siècle et demi la problématique des mémorisations par les lieux. Elle conditionne celle des contenus : que retenir, qu'oublier? C'est en cela que l'on peut parler de ce processus comme d'une «localisation». Il faut bien comprendre ce que veut ainsi dire le mot: non pas l'endroit, la localisation au sens des coordonnées géographiques, mais la localisation au sens de mise en lieu, dans la même idée que l'on parle de mise en mots, de mise en acte ou de mise en image, etc.

Ce processus de localisation participe de la logique beaucoup plus générale de production des espaces habités géographiques. L'espace brut est ainsi informé, soit à la fois porteur d'informations et agencé en lieux et territoires du monde (Lazzarotti 2006). Ce processus passe par des discours, tous les types de discours. Et par les pratiques : ce que les «hommes font et comment ils le font", pour reprendre l'expression de Michel Foucault (Dekens 2004: 22), quitte à y ajouter «où ils le font». Ainsi, l'analyse géographique ne prend pas à la lettre l'expression « lieux de mémoire », du moins telle qu'elle a pu être utilisée par la somme de Pierre Nora dans laquelle l'usage métaphorique se mêle, quitte à s'y confondre, au sens matériel. Car ce qui importe au point de constituer la singularité de l'entrée géographique dans le champ des mémoires est ce processus de localisation, de mise en lieu qui montre en matérialisant ce qui est retenu du passé et, à l'occasion, interprété. Le processus dynamique est ainsi celui d'une production conjointe, en l'occurrence l'écriture simultanée des mémoires et des lieux.

\section{Mémoriser}

11 Privilégier cette approche géographique, c'est donc reprendre et déployer la distinction formulée dès la Révolution française entre deux processus de mémorisation : mémoire monumentale et mémoire patrimoniale. C'est aussi l'occasion de clarifier, par le rapport à l'espace, les différences entre les deux notions. Comment?

\section{La mémoire monumentale, une mémoire dite}

12 Dans ce débat, le processus monumental s'impose assez vite comme modalité de mémorisation dominante. Il est défini par le fait que ce sont les discours des érudits et des savants qui désignent les objets dignes d'être retenus. Parmi ces acteurs, on trouve des écrivains - Victor Hugo en tête, mais aussi Prosper Mérimée -, des artistes, mais aussi des scientifiques et des architectes, Eugène Viollet-le-Duc s'imposant comme l'un des premiers à réfléchir au sens, si ce n'est aux techniques, de restauration. Souvent liés les uns aux autres et à l'occasion associés au pouvoir, ils assoient leur légitimité en investissant les administrations étatiques. Et ainsi, la Commission des monuments historiques, créée en 1837, inventorie, classe, protège : elle dicte. Et dessine les repères historiques et géographiques du pays en recomposition.

13 Cette France est celle de l'État-Nation. Elle est celle de l'enracinement qui, dans la logique toute lamarckienne de l'harmonie entre un sol et ses habitants, est sensé 
définir et affirmer le modèle politique dominant : celui du paysan sédentaire, attaché à un lieu unique où il naît, travaille, se reproduit et meurt, et qu'il ne quitte que pour aller combattre d'autres paysans-soldats qui considèrent défendre une cause identique. Mais de l'autre côté de la frontière.

À la veille de la Première Guerre mondiale, le modèle s'est définitivement imposé. Pour autant qu'on puisse en juger, la cause monumentale est intériorisée, à la manière d'un habitus. La loi du 31 décembre 1913 l'inscrit dans les marbres de la République tandis que la grande histoire de France d'Ernest Lavisse, soutenue par le géographe Paul Vidal de la Blache(1903) en reprend, implicitement, les termes idéologiques : "L'histoire d'un peuple est indissociable de la contrée qu'il habite." Les destructions des années 1914-1918 ne feront qu'en réactiver l'énergie quand, au sortir de l'horrible conflit, les inscriptions sur la liste des Monuments historiques et les restaurations qui vont avec, à commencer par celle de la cathédrale de Reims, se feront de plus en plus nombreuses.

Affiche de propagande militaire, 1915.

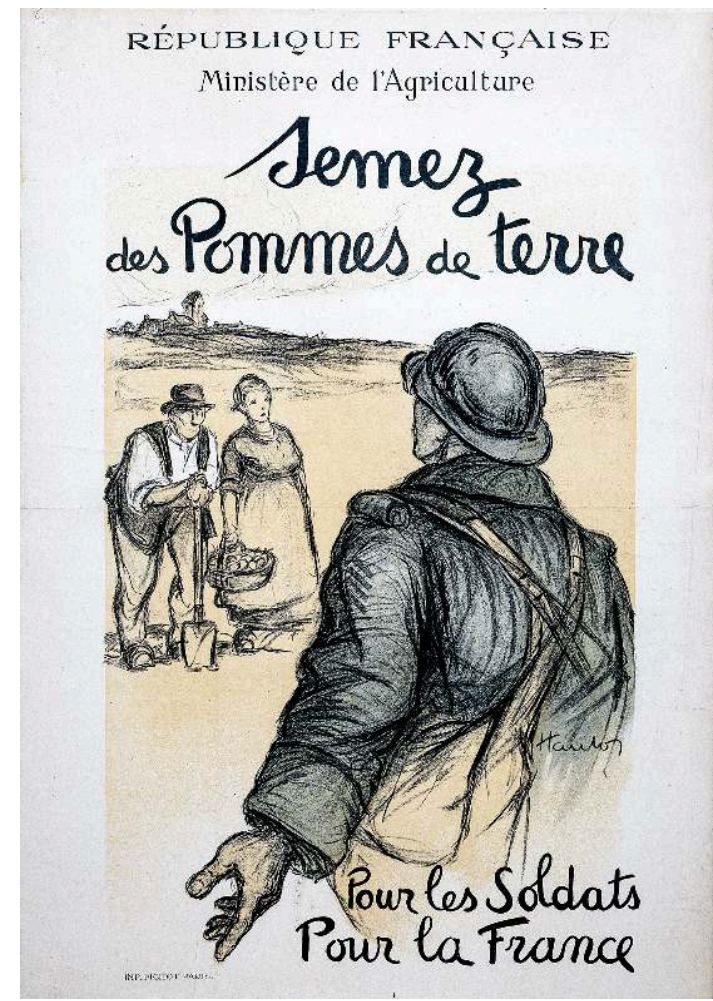

Fertiliser le sol et le défendre : idéal politique du paysan-soldat.

(c) Pascal Segrette / cliché musée de l'Armée, dist. RMN-Grand Palais.

\section{La mémoire patrimoniale, une mémoire par les autres}

Les horreurs de la Seconde Guerre mondiale, en général, et celles d'Auschwitz, en particulier, auraient-elles poussées à la tabula rasa? On pourrait le penser tant la fin des années 1940 et les années 1950 sont celles d'un « trou mémoriel ». De fait, ce n'est qu'au cours des années 1960 que le passé redevient une question sociétale (Choay 2009). Mais en même temps, il le fait selon d'autres modalités et le changement n'est pas que 
quantitatif. Il aboutit, en novembre 1972, à la signature de la Convention du patrimoine mondial : autre mémoire, autre mot?

Analysée au prisme de sa principale mise en œuvre, la mémoire patrimoniale se distingue facilement d'une aînée qui, sans disparaître, perd son monopole. Le changement est d'abord d'échelle. Il marque le passage d'une mémoire nationale à une mémoire internationale. Dans le mouvement, sont réunis sous le même vocable, et dans un premier temps pour en maintenir les différences, les deux monumentalités. Le patrimoine mondial est culturel ou naturel. Autre transformation, et pas des moindres : les discours ne sont plus les sources exclusives du processus de mémorisation. Les regards des autres et, parmi eux donc, ceux des touristes, participent à la reconnaissance mémorielle. Et il est aisé de le reconnaître. Les grands sites du patrimoine sont aussi des grands sites touristiques. Et les pays importants par le nombre de leurs sites sont aussi des pays très visités par les touristes internationaux. Les chiffres parlent alors d'eux-mêmes: de fait, patrimoine et tourisme sont coconstitués (Lazzarotti 2000). Bien que longtemps considérés comme une "menace", théorisée tant bien que mal par la notion de "capacité de charge touristique », les touristes s'imposent plutôt comme producteurs et promoteurs de mémoires. Par leur présence, par leur regard, ils valident, confirment, légitiment les objets ainsi patrimonialisés. Dès lors, aussi bien par l'institution du patrimoine mondial que par les pratiques des uns et des autres, touristes ou non, la mémoire patrimoniale est une mémoire élue. En tant que telle, elle est, plus évidemment encore que son précédent modèle, une mémoire politique.

Entretenir les monuments : guerre ou tourisme?

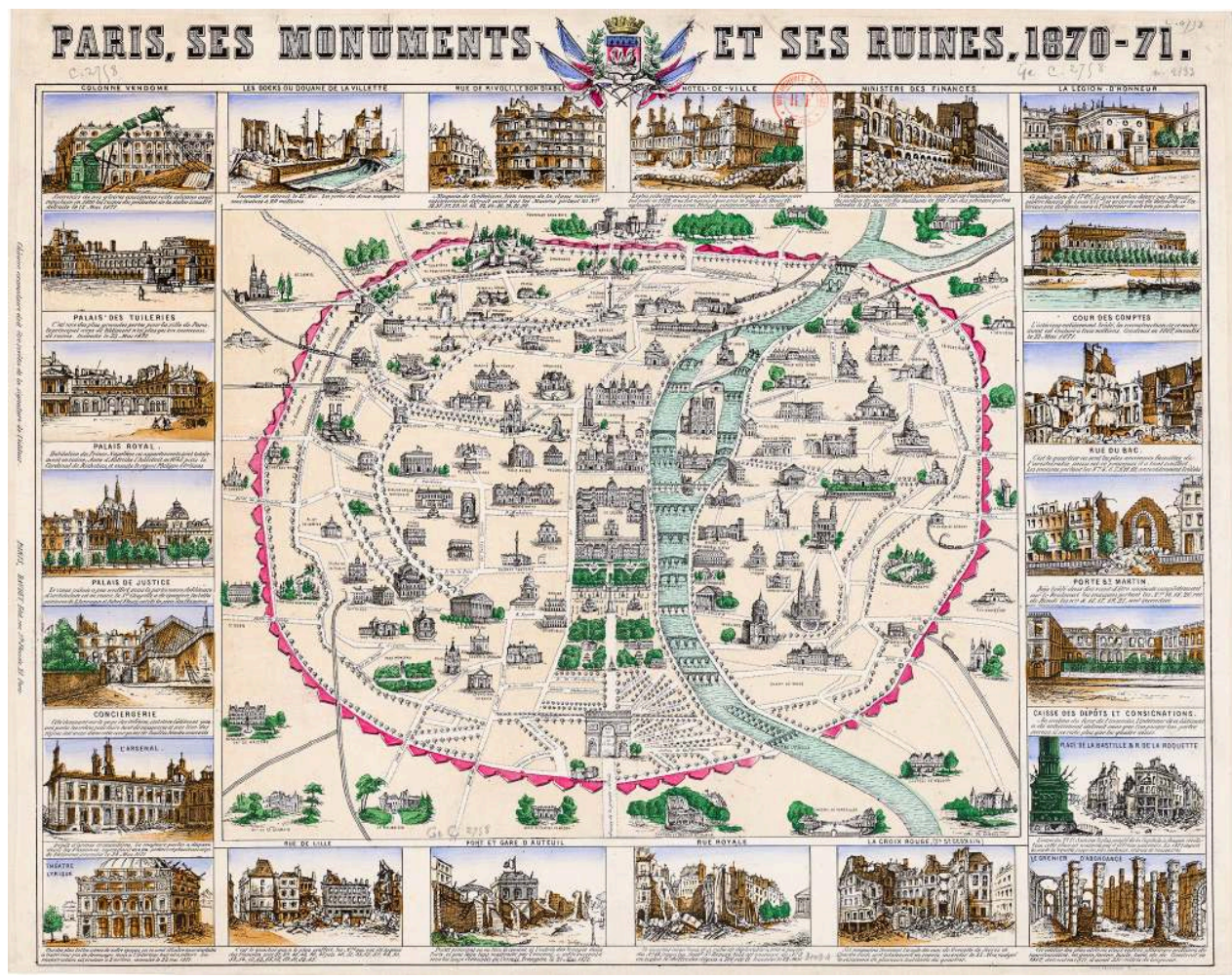

Plan touristique : Paris, ses monuments et ses ruines, Baudel éditeur, 1870-1871.

(c) Bibliothèque nationale de France. 
17 L'institution en tint compte. Pour être légitimement "mondiales ", les mémoires patrimoniales se doivent d'être équitablement partagées dans le monde. Quitte, pour cela, à changer la définition même du patrimoine et avec elle les critères de sa reconnaissance. Ainsi, si ceux des moments fondateurs des années 1970 sont encore très inspirés par les débats monumentaux de l'Europe, la «stratégie globale » de 1994, prolongée par les conclusions de la dix-huitième session du Comité du patrimoine mondial tenue à Phuket, imprime une inflexion « anthropologique » à la définition. Non localisés au sens strict du terme, des faits sociétaux peuvent désormais être listés. On pense au tango argentin (inscrit en 2009) ou à l'acupuncture (inscrite en 2010). Une autre modification est introduite à la session de Budapest, en 2002. En associant Patrimoine mondial et développement durable, c'est une liste unique de dix critères qui, quels que soient les cas, décident de l'authenticité et de l'intégrité qui font la « valeur universelle exceptionnelle » des sites proposés. Les mémoires patrimoniales se rangent alors résolument dans la catégorie des biens à transmettre alors que de nouvelles catégories font leur entrée, celle des « itinéraires culturels », par exemple et entre autres, qui élargissent d'autant les possibilités de classement.

Les autres, visiteurs, à l'occasion touristes, sont donc finalement reconnus comme partie prenante du processus de mémorisation. En tant que tels, ils sont intégrés aux réflexions. Les références doctrinales orientant l'articulation patrimoine-tourisme varient. Au début des années 2000, le rapport d'Arthur Pedersen (2002) dote l'Unesco d'une nouvelle grille de lecture et d'actions. Elle est celle des « limites du changement acceptable ». On en comprend vite le sens : ce n'est pas le nombre de touristes qui, en soit, fait problème, mais le comportement qui peut être celui de quelques-uns, très peu même. Car un seul malveillant peut jeter le doute sur des milliers de visiteurs précautionneux. Plus globalement, la nouvelle doctrine se fait plus souple, reconnaissant que les effets du tourisme doivent être considérés au cas par cas et selon des critères plus relatifs qu'absolus. Du reste, l'observation courante le confirme encore. Le rapport aux touristes n'est pas le même à Lijiang, site chinois du Patrimoine mondial le jour et, disons-le simplement, un peu bordel la nuit, qu'au Mont SaintMichel. 
Les touristes, sauveurs d'une Tour Eiffel construite pour être détruite.

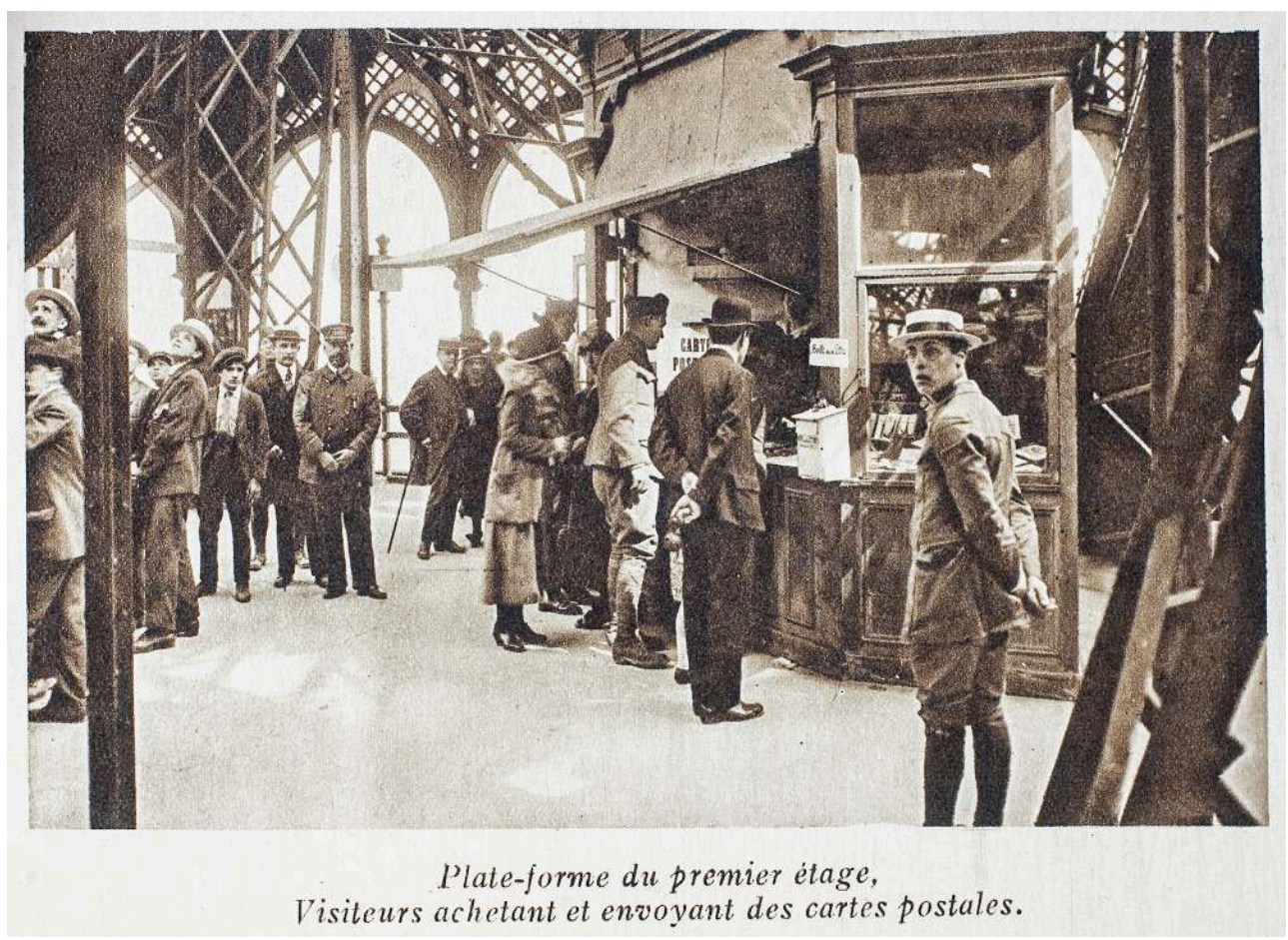

La Tour Eiffel, plateforme du premier étage. Visiteurs achetant et envoyant des cartes postales, Neurdein Frères (1863-1915).

(c) Patrice Schmidt / musée d'Orsay, dist. RMN-Grand Palais.

\section{Mémoires-Monde}

Si Val d'Europe, ville bâtie dans la sphère de Disneyland Paris, date du début des années 1990, le choix de son vocabulaire architectural évoque indiscutablement la ville haussmannienne. Voilà donc le cas d'un lieu construit comme déjà mémoriel. Ailleurs : il n'est qu'à flâner dans la rue Champlain de Québec, ou bien dans celle de Bercy-Village - attention aux rails de chemin de fer -, ou bien le long de la rive droite de la Tamise à South Bank ou bien dans une partie restaurée de la concession française de Shanghai, justement nommée Xintiandi (新天地), littéralement «Nouveau Monde». Pris au quatre coins du monde - et encore n'avons-nous rien dit de lieux comme Dubaï-, tous ces lieux, au-delà de leurs allures différentes, ont entre autres points communs d'afficher un fort rapport au passé. De ce point de vue, ils sont bien mémoriels. Mais alors, et pour autant, ils ne ressemblent en rien aux mémoires monumentales ou aux mémoires patrimoniales. Il convient donc bien d'en tenir compte pour s'interroger : et si ces deux régimes mémoriels, de fait initiés par la Révolution française et déployés au cours de deux siècles précédents le nôtre, n'épuisaient plus aujourd'hui et les processus et les paysages des lieux mémoriels autrement dit l'éventail contemporain des relations entre lieux et mémoires? 
Bercy village : local parce que mondial, mondial parce que local.

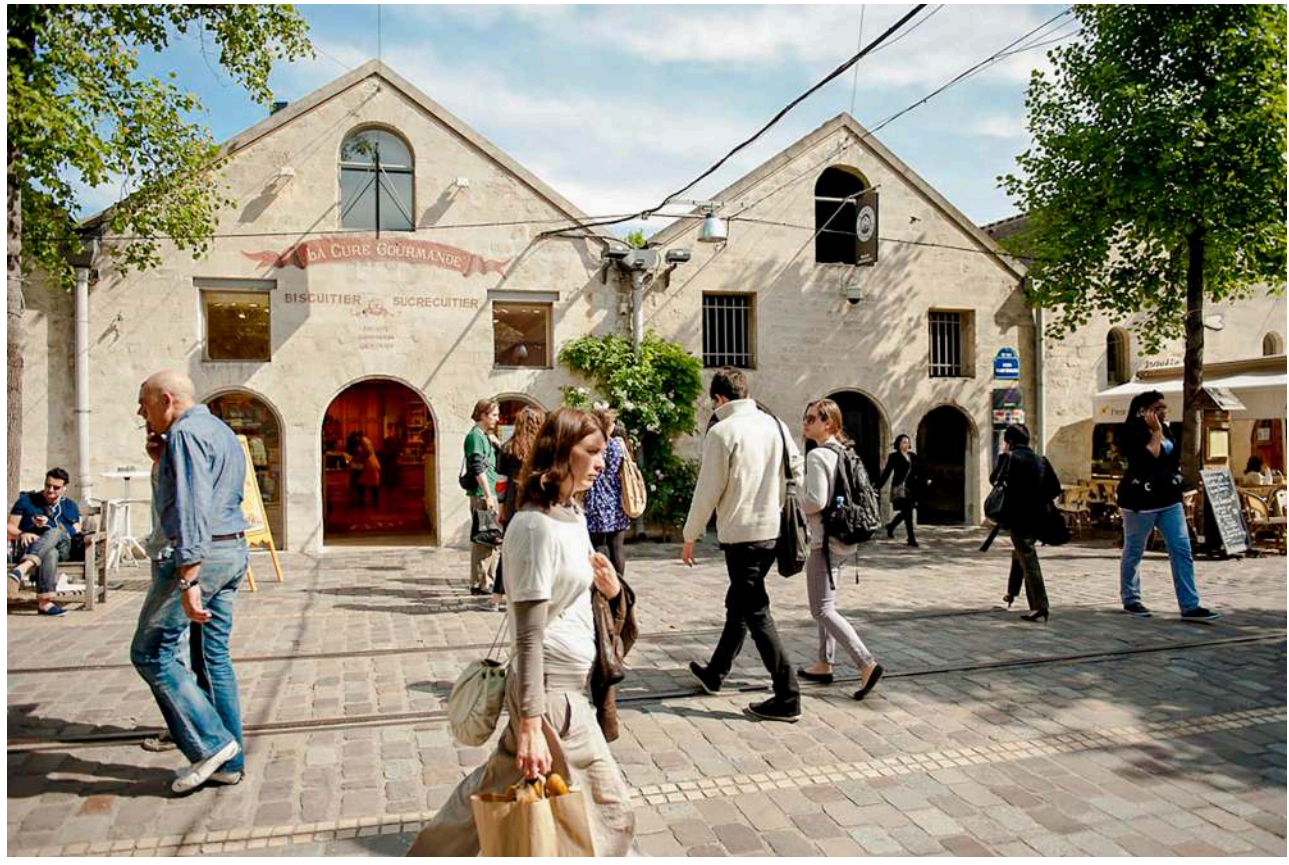

(c) Luc Valigny / Bercy village.

20 L'expérience de Portmeirion, au Pays de Galles, constitue sans doute l'un des lieux génériques de cette catégorie. Depuis le moment de sa création dans les années 1920, des architectures inspirées de la Renaissance italienne y côtoient des bâtiments du $\mathrm{XIX}^{\mathrm{e}}$ siècle, authentiques mais déplacés. Le lieu rompt ainsi avec les déterminismes historiques et géographiques qui fondent, qualifient et légitiment les «régimes d'authenticité " (Morisset 2009) des mémoires monumentales et patrimoniales. L'histoire ne s'est pas déroulée là où elle est montrée. Et que dire de l'agencement des lieux, dès lors que l'Angleterre s'organise autour d'une place aux statues asiatiques? Ainsi, à Portmeirion, pas plus que la géographie n'est faite de lieux en continuité, le présent n'est le prolongement linéaire du passé. Il l'englobe à la manière d'un « présent global » qui, de manière réticulaire, est fait de toutes les époques et de tous les lieux. Les principes de la linéarité temporelle et de la continuité spatiale volent en éclat. Cette spécificité n'est pas la seule que partagent, peu ou prou, tous ces lieux.

21 À Xintiandi, les shikumen, résidences habituelles de populations aisées, mais à peine, constituent l'essentiel du bâti revalorisé. À Bercy, quelques anciens dépôts à vin assurent l'armature du tout. Et les petites maisons de la Place Royale et de ses environs ne rappellent rien d'autre que la vie ordinaire. Ici, rien n'a véritablement de valeur universelle exceptionnelle: de telles mémoires valorisent des rappels du quotidien, banal et courant, des gens - toutes choses que les monuments et le patrimoine ignorent.

Troisième caractère: tous ces lieux sont associés à des équipements culturels, en général à un ou plusieurs musées. Souvent thématisées sur des histoires populaires, tel le musée des arts forains à Bercy, ils alimentent l'activité culturelle du lieu et le valorisent symboliquement, et au-delà.

Car le projet de rentabilité financière est au cœur de ces logiques locales. Les investissements sont privés. L'argent sert à restaurer le tout alors que l'investissement 
soutient l'ouverture de très nombreuses boutiques. Le plus souvent, celles-ci commercialisent des produits d'apparence artisanale et d'allure plutôt locale. Cela va parfois plus loin, comme le long du Grand Canal à Hangzhou où un ensemble d'ateliers artisanaux ont élu domicile pour fabriquer de petits objets à voir et à vendre. De même, de nombreux restaurants confirment l'importance des visiteurs, tous clients. Car là est le ressort de tous ces lieux-boutiques, à l'occasion actualisation d'anciennes traditions, comme c'est le cas des nombreuses rues-boutiques en Chine. Les affaires sont au cœur de la production de ces lieux, et l'argument mémoriel est l'un de leurs soutiens. Il y a donc, bien sûr, les commerces, mais il y a aussi, portés par la valorisation symbolique et iconographique d'une offre proposée comme culturelle, d'importants programmes immobiliers. Il est dévolu à ces derniers d'assurer la couverture et la rentabilité financières du projet. Si tous les ressorts de l'économie présentielle (Davezies 2008) y sont posés, ils ne sont pas les seules cordes de l'arc économique qui se tend.

Mémoires-Monde : visiter, consommer.

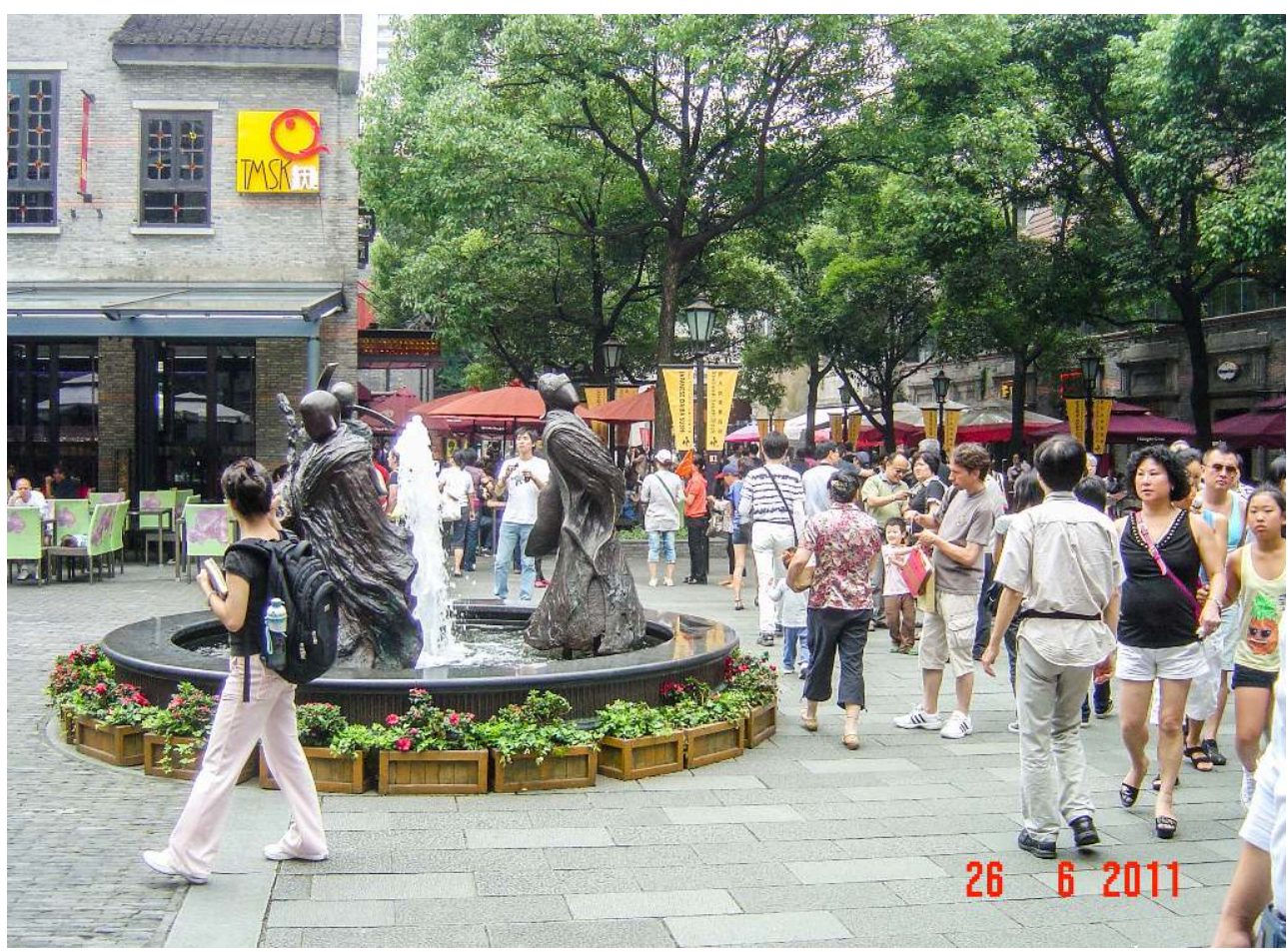

Rue-boutique, avec ses produits « artisanaux »; un équipement culturel (le musée du Shikoumen, résidence des « classes moyennes du début du XXe siècle) ; des restaurants ; un arrière-plan de programme immobiliers : Xintiandi Shanghai.

(c) Olivier Lazzarotti.

De tels lieux mémoriels se présentent ainsi dans une double dimension. Ils sont d'abord tout à fait locaux. Les traditions, les architectures, les productions relèvent d'une silhouette locale, quand bien même une partie d'entre elles viendrait de plus loin. À Xintiandi, pas de doute, on est en Chine ; rue Champlain, pas de doute, on est à Québec ; à Bercy-village, pas de doute, on est à Paris, etc. Cet ancrage local est on ne peut mieux servi, alimenté, promu par l'argument mémoriel. Mais en même temps, ils sont mondiaux. Tous ces lieux sont ouverts, facilement visitables : quiconque en a pratiqué un les a pratiqués tous. Ils sont ouverts aussi parce que ces visiteurs, des touristes venus du plus loin aux usagers les plus proches, sont les moteurs du système 
économique qui les fondent. C'est la raison pour laquelle, plutôt que de torturer la notion de patrimoine, j'ai fait le choix de l'abandonner pour proposer de qualifier ces lieux de «mémoires-Monde» (Lazzarotti 2012). De multiples manières, ces lieux, mémoriels ne serait-ce que dans leur vocabulaire architectural, se distinguent des monuments et des patrimoines. Ils sont ceux d'une mémoire du quotidien, d'une mémoire courante des habitants usuels, banals pourrait-on dire. Il en est ainsi des entrepôts de vins de Bercy-village. Portées par des investisseurs privés, la rentabilité est l'un de leurs moteurs : rentabilité d'une rue prenant des allures de rue-boutiques; rentabilité des programmes immobiliers adjacents et qui profitent de la plus-value symbolique de l'aménagement. La présence des visiteurs, des touristes venus du bout du monde aussi bien que des badauds venus de trois rues voisines, alimente ainsi les rouages d'une économie présentielle, à l'occasion rehaussée d'un équipement culturel, le plus souvent sous forme de musée. Tout cela combiné fait que ces lieux sont locaux parce que mondiaux, mondiaux parce que locaux. Tous différents mais toujours identiques - et même d'autant plus différents qu'ils sont identiques.

Lieux du Monde - avec un « $M$ » majuscule pour désigner une dimension à la fois originale et unique associée à la géographie contemporaine (Lussault 2013, par exemple) -, les mémoires-Monde constituent le versant temporel de la globalisation contemporaine : des temps liés, des temps reliés. Un réseau de temps, hors de toute continuité linéaire.

\section{Écrire}

Les relations entre mémoires et espace sont marquées par une diversification des processus de mémorisation. Au-delà du contenu mémoriel lui-même, ils relèvent des grands mécanismes de production des espaces habités géographiques.

Par les discours - tous les discours, y compris les discours non verbaux -, mais aussi par les pratiques - y compris le tourisme -, les mémoires forment et informent en même temps les lieux et les territoires du monde. Travaillant ainsi la matière de la Terre, les sociétés trient leurs mémoires et choisissent leurs oublis, de telle sorte que l'on peut parler de cette dynamique comme d'un procédé d'écriture, ne serait-ce qu'en prenant en première analyse la définition qu'en donne Jacques Derrida (1967: 65), l'« inscription et institution durable d'un signe ». Dans cette perspective, les gestes de la géo-graphie y trouvent leur sens, à commencer par les trois acceptions reconnues du mot grec « graphō » : érafler, dessiner, écrire (Irigoin 1982).

\section{Symboliser}

Les discours des savants, des chercheurs, des spécialistes, des artistes, etc. relayés par les aménageurs en tout genre (architectes, restaurateurs, designers...) et par l'habileté des artisans et divers corps de métiers techniques, participent à l'écriture géographique des mémoires. Au sens le plus technique, ils en sont les fabricants. Ils en modèlent les matières et les agencent. De ce point de vue, ces producteurs sont des décideurs. Pas totalement pourtant.

Car ces mémoires n'ont d'existence que du fait d'être lues, que par l'action de leurs lectrices et de leurs lecteurs. Ces derniers participent de deux manières. Par leur présence d'abord. Être dans un lieu mémoriel, c'est lui donner vie, le reconnaître et le 
valider comme tel, à l'occasion le changer. De ce point de vue, la présence des autres, touristes ou non, participe pleinement du processus de mémorisation. Si leur rôle est réduit dans le cas des mémoires monumentales, il est plein et entier dans le cas des mémoires patrimoniales dont ils constituent une marque de fabrique. Et c'est encore le cas pour les mémoires-Monde.

Mais la présence des habitantes et habitants intervient aussi de façon plus qualitative. La portée mémorielle des lieux en dépend. Le second mode de participation mobilise les représentations, les inconscients, les idéologies, les savoirs et les ignorances de ces lectrices et lecteurs. C'est par les systèmes d'interprétation dont ils sont porteurs et par les jeux d'encodage et de décodage qui les sous-tendent que fait sens la "différance »pour reprendre la notion de Derrida (Le Monde 1984) -, autrement dit qu'est comprise la séparation temporelle entre ce qui est vu et le ou les événements qui font mémoire : ce qui semble le plus présent n'est-il pas déjà passé ? Et ce qui semble passé n'est-il pas bien présent?

31 La localisation des mémoires transforme la matière dont est faite la terre, l'informe en la mettant en forme - une forme qui, parce qu'elle est interprétée, fait signe. Un signe qui puise son énergie communicative à la source des symboles : la géo-graphie est bien écriture.

\section{Dire}

Ici émerge la seconde particularité de cette singulière écriture. Faisant de la Terre aussi bien que de ses habitants des signes, elle ne grave pas des mots. Langage à part entière, elle ne fige pas un verbe parlé. Elle ne fait rien entendre. En revanche, matérialisant le passé, elle rend visible. Elle montre. Mises en lieux, palpables et sensibles, les mémoires sont ainsi pleinement associées à cette humaine expérience du monde, l'expérience géographique qui est celle de ses habitantes et habitants. Dès lors, que montrent les lieux ? Que contiennent-ils ? Plus particulièrement, que retiennent-ils lorsqu'ils ils sont pleins de mémoires? Que dit ce langage géographique ? De quoi informent les espaces habités géographiques?

Retenons, d'abord, qu'ils affichent ce que les sociétés veulent donner à voir d'ellesmêmes, convoquant ainsi l'autre lecteur - de qui s'agit-il ?-, ce premier auteur des lieux, en qualité de principal témoin. Mettre en avant tel ou tel élément du passé, c'est se référencer en choisissant les faits qui feront une Histoire. Ainsi s'élabore un processus d'identification à l'adresse de ces autres qui, le confirmant ou non, participeront peu ou prou à sa validation. Cela dit, ce processus d'identification engage aussi un processus de légitimation: les détenteurs de la mémoire seraient-ils les habitants légitimes d'un lieu? Ou bien voudraient-ils en convaincre les autres? Il conviendrait sans doute de formuler autrement l'interrogation: comment des habitants trouvent-ils dans le passé les argument de leurs légitimes présences. Et pourquoi dans ce passé, qui est souvent présenté comme celui du " premier arrivant »?

Et encore : ce que dit l'écriture géo-graphique dépasse la référence à tel ou tel passé. En produisant un référentiel mémoriel c'est l'agencement et, partant, l'ordre des espaces habités géographiques, leur organisation politique, entre autres celle des interrelations humaines, qui s'y joue. Car au-delà du tri entre ce qui doit être retenu et ce qui va voire doit - être oublié, c'est toute la mesure du temps et, avec elle, sa valeur, ses hiérarchies autrement dit l'ensemble de sa construction, qui est convoquée, à l'occasion 
débattue. Et parce que l'espace habité géographique ainsi formé et informé par les mémoires n'est pas seulement le paysage décoratif des vies humaines, une telle construction met directement en cause la cohabitation locale: les interrelations humaines dans leur dimension politique.

Dès lors, la portée des conflits mémoriels se révèle comme autant de conflits sociétaux. Ils peuvent être interprétatifs : que voir à Hiroshima? Un ensemble pour la paix ou le résultat d'une politique impérialiste catastrophique pour tous? Ils peuvent être référentiels : s'il s'agit de restaurer, que restaurer, que détruire? Mais aussi comment restaurer, pour quoi et pour qui ? Pour accompagner un changement d'habitants, faisant sortir d'un lieu les uns et entrer les autres? Ces conflits peuvent aussi impliquer l'argument de légitimité. Par exemple, quelle place, dans l'espace public, pour les quelques restes du mur du ghetto de Varsovie et, au-delà, quelle place pour la présence juive en Pologne?

Berlin, Allemagne, Europe : quelle place pour quelle histoire?

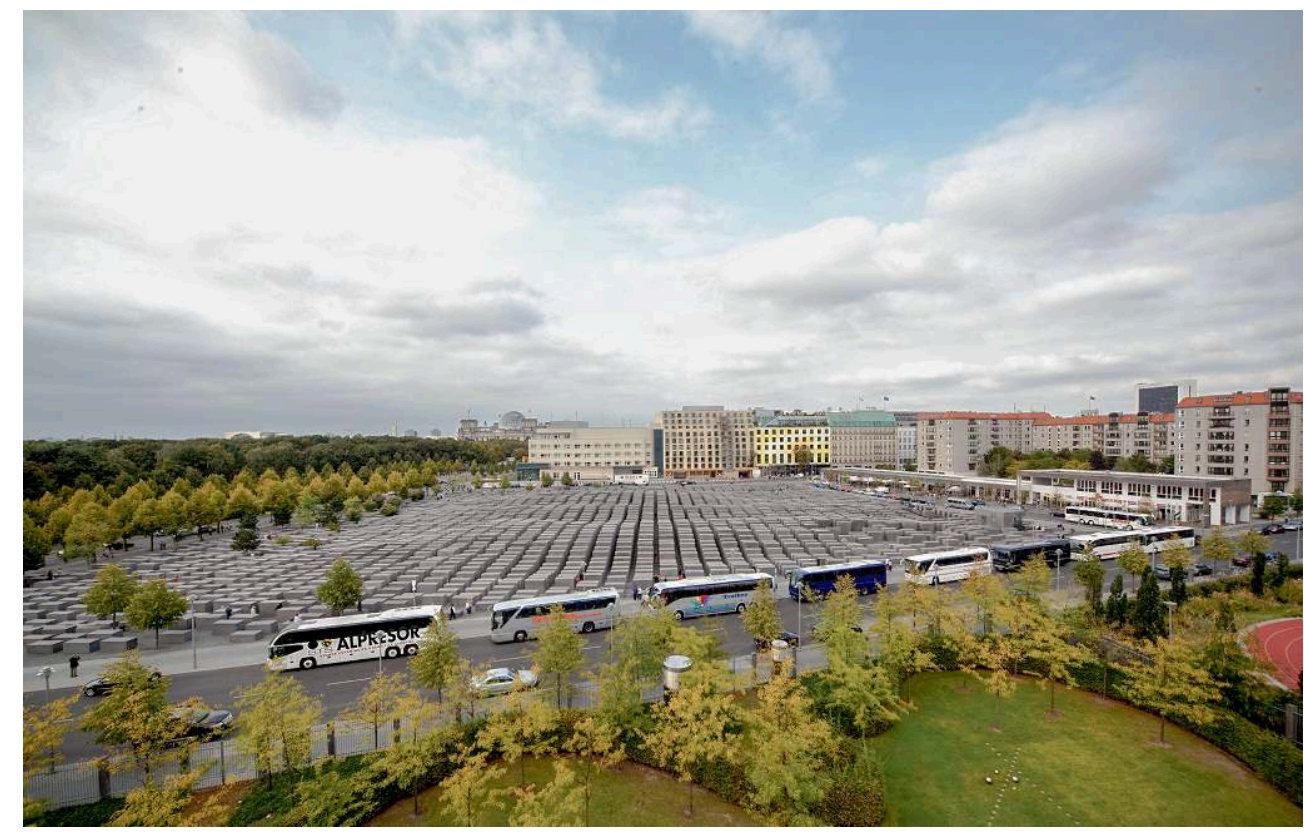

Sur des terrains libérés par la destruction du mur, le Mémorial aux juifs assassinés d'Europe. Et le tourisme, pacificateur du lieu?

(c) Marko Priske / cliché Mémorial aux juifs assassinés d'Europe.

De manière particulière lorsqu'il s'agit de mémorisation, mais d'une manière qui réfléchit celle de tous les espaces habités géographiques, les lieux et territoire du monde conditionnent, les inventant ou les reproduisant, les contestant à l'occasion pour les réorganiser, les fonctionnements et les dynamiques des relations sociales de celles et ceux qui les habitent. De cette manière, ils ne sont pas seulement un témoignage, un reste de ce qui a été ou un moule de ce qui doit être, mais bien plus encore, dans son actuel présent, un de ses enjeux. Le résultat et les processus s'y confondent à l'occasion. Et les régimes mémoriels, qui participent à en formuler la conception générale, réfléchissent pleinement les enjeux politiques qui s'y trament. Sur ce point, Joseph Morsel (2016) ne dit rien d'autre: «Bref, le "Patrimoine" ne dit rien sur les objets concernés, mais sur notre société. » 


\section{Que retenir des mémoires?} de son entrée. En posant la question des relations entre espaces et mémoires, elle l'analyse sous l'angle d'un processus de mémorisation. Étudier l'inscription des mémoires dans les lieux, la considérer comme "régime de mémorisation » pour en analyser les acteurs et les moyens, c'est prendre le problème sous l'angle dynamique d'un processus d'écriture, géographique s'il en est.

41 3. Il existe un lien entre régimes de mémorisations, écriture mémorielle des lieux et contenu des mémoires. On observe du reste que chaque changement de régime conduit à mémoriser d'autres lieux, d'autres temps, d'autres formes. Les débats sur les acteurs des mémorisations légitimes ne sont pas uniquement «techniques», si l'on peut dire. Ils impliquent aussi ce qui est retenu, ou oublié. De ce point de vue, on peut s'inspirer des remarques de Louis Althusser (2005), pour suggérer que les mémoires dominantes sont malgré tout celles des classes dominantes. De ce point de vue, la monumentalisation et la symbolisation de l'écriture mémorielle géographique peut très bien agir comme processus de «naturalisation»: produire l'oubli, l'oubli des conditions de production, l'oubli même qu'il y eut production. Et quand. Et que rien n'est donné, pas plus le passé que tout le reste.

4. Mise en perspective dans le temps et les lieux, l'écriture géographique des mémoires fait apparaître des différences selon les moments et les sociétés. Toutes les époques ne sont pas également mémorielles. Et celles qui le sont ne le sont pas de la même manière. Dès lors, il est possible de remarquer que les périodes d'abondance mémorielle correspondent à des moments de forts changements sociétaux. Et si les mémoires, au lieu de figer le passé étaient plutôt, au moment du renouvellement de leur régime, l'un des premiers matériaux d'expérimentation et de construction de temps nouveaux ? Cela ne signifierait-il pas que la relecture du passé, avant que d'être, peut-être, une position conservatrice, accompagne les premiers gestes d'un présent renouvelé?

43 5. "L'espace est une réalité qui dure ", soutenait Maurice Halbwachs (1997: 209), suggérant également que "la société [y] immobilise une partie d'elle-même " (ibid.: 235). Ce faisant, Halbwachs a-t-il été le premier à évoquer la possibilité d'une théorie spatiale - géographique ? - des mémoires. Cela mérite, aujourd'hui, d'être retenu. Mais aussi prolongé. À l'ère du Monde et de ses sociétés à habitants mobiles (Lussault 2013), 
on peut légitimement s'interroger : la localisation des mémoires n'est-elle pas l'un des moyens dont les tenants des lieux disposent pour les construire en mobilisant ce qu'ils ont de non-délocalisable? Les faits passés, alors même que l'histoire positive n'est en rien convoquée dans cette vérité, ne sont-ils pas les meilleurs facteurs de la production d'une « rente de monopole» (Harvey 2001). Pour qui veut voir la Tour Eiffel, quel autre moyen que de venir à Paris? Et qui veut faire son marché dans une ambiance typiquement chinoise, mais rendue accessible à tous, n'a qu'à se rendre à Xintiandi, etc. On comprend ainsi le recours aux mémoires, aussi bien que la spécificité des processus de mémorisation. Ils comptent parmi les arguments les plus efficaces de la singularisation des lieux. Comprendre : si l'on définit l'actuelle mondialisation comme celle des lieux liés et reliés, liaisons qui accompagnent la mobilisation généralisée des habitants, il faut aussi reconnaître que les lieux se trouvent dans une situation pour le moins inédite. Avec la fin des assignations de chacun, et avec un nombre croissant de personnes de plus en plus mobiles, les lieux se retrouvent en concurrence les uns avec les autres. Se singulariser est un moyen pour mettre en avant la "rente de monopole " sensée donner au plus grand nombre l'envie de venir, et alimenter ainsi les dynamiques des économies présentielles (Davezies 2008). Les processus mémoriels contemporains font ainsi pleinement partie de ces nouveaux processus de valorisation de lieux qui, de gré ou de force, choisissant de ne pas cultiver l'exploitation des "ressources" naturelles, cherchent à se promouvoir en combinant notoriété et connexion au Monde. De sorte que ces cinq leçons conduisent à un même projet : travailler sur et avec les mémoires, c'est travailler sur les pensées contemporaines de la science géographique du Monde contemporain.

\section{BIBLIOGRAPHIE}

ALTHUSSER Louis, 2005, Pour Marx, Paris, La Découverte, coll. « La Découverte-poche. Sciences humaines et sociales ».

ASHWORTH Gregory, GRAHAM Brian \& TURNBRIDGE John E. (dir.), 2000, A geography of heritage. Power, culture and economy, Londres, Arnold.

BERGSON Henri, 1990 [1896], Matière et Mémoire. Essai sur la relation du corps à l'esprit, Paris, PUF, coll. « Quadrige ».

BOURDIN Alain, 1984, Le Patrimoine réinventé, Paris, PUF, coll. « Espace et liberté ».

CANDAU Joël, 1996, Anthropologie de la mémoire, Paris, PUF, coll. « Que sais-je?».

CHASTEL André, 1994, Architecture et Patrimoine. Choix de chroniques du journal « Le Monde », Paris, Imprimerie nationale / Inventaire général des monuments et des richesses artistiques de la France.

CHOAY Françoise, 1991, L'Allégorie du patrimoine, Paris, Éditions du Seuil, coll. « La couleur des idées". 
CHOAY Françoise, 2009, Le Patrimoine en questions. Anthologie pour un combat, Paris, Éditions du Seuil, coll. « La couleur des idées ».

DAVEZIEs Laurent, 2008, La République des territoires. La circulation invisible des richesses, Paris, Éditions du Seuil, coll. « La République des idées ».

DEKENS Olivier, 2004, Qu'est-ce que les Lumières? Michel Foucault, Paris, Bréal, coll. « La Philothèque ", 2004.

DEPRAZ Samuel, 2008, Géographie des espaces naturels protégés. Genèse, principe et enjeux territoriaux, Paris, Armand Colin, coll. « U. Géographie ».

DERRIDA Jacques, 1967, De la grammatologie, Paris, Les Éditions de Minuit, coll. «Critique ».

FARMER Sarah, 1994, Oradour, arrêt sur mémoire, Paris, Calmann-Lévy, coll. « Essai histoire ».

GREFFE Xavier, 2003, La Valorisation économique du patrimoine, Paris, La Documentation française / ministère de la Culture et de la Communication, coll. «Questions de culture ».

HALBWACHS Maurice, 1994 [1925], Les Cadres sociaux de la mémoire, Paris, Albin Michel, coll. «Bibliothèque de l'évolution de l'humanité ».

HALBWACHS Maurice, 1997 [1950], La Mémoire collective, Paris, Albin Michel, coll. « Bibliothèque de l'évolution de l'humanité ».

HARVeY David, 2001, Géographie de la domination, Paris, Les Prairies ordinaires, coll. « Pensercroiser ».

HÉRITIER Stéphane (dir.), 2009,

Nature et patrimoine au service de la gestion durable des territoires, Paris, Publications Missions ressources et compétences technologiques (CNRS), coll. « Intégrations des savoirs et des savoirfaire $»$.

HERTzog Anne, 2011, « Les géographes et le patrimoine », EchoGéo [en ligne], n 18, http:// echogeo.revues.org/12840 [lien valide en septembre 2018].

IRIGoIN Jean, 1982, « Les Grecs et l'écriture. Quelques jalons historiques », Corps Écrit, n 1, «L'écriture », p. 31-38.

JEUDY Henri-Pierre, 2001, La Machinerie patrimoniale, Paris, Sens \& Tonka, coll. « 10/vingt : essai ». LAZZAROTTI Olivier, 2000, « Patrimoine et tourisme : un couple de la mondialisation », Mappemonde, $\mathrm{n}^{\circ} 1$, p. 12-16.

LAZZAROTTI Olivier, 2006, Habiter. La condition géographique, Paris, Belin, coll. « Mappemonde ». LAZZARotTI Olivier, 2011, Patrimoine et Tourisme. Histoire, lieux, acteurs, enjeux, Paris, Belin, coll. « Belin sup. Tourisme».

LAzZARoTti Olivier, 2012, Des lieux pour mémoires. Monument, patrimoine et mémoires-Monde, Paris, Armand Colin, coll. « Le temps des idées ».

LE MONDE, 1984, Entretiens avec « Le Monde », Paris, La Découverte, p. 78-90.

Lussault Michel, 2013, L'Avènement du monde. Essai sur l'habitation humaine de la Terre, Paris, Éditions du Seuil, coll. « La couleur des idées».

MORISSET Lucie K., 2009, Des régimes d'authenticité. Essai sur la mémoire patrimoniale, Rennes, Presses universitaires de Rennes, coll. « Art \& société ». 
MORSEL Joseph, 2016, « Traces ? Quelles traces ? Réflexions pour une histoire non passéiste », Revue historique, vol. 4, $\mathrm{n}^{\circ}$ 680, p. 859. Disponible en ligne, http://www.cairn.info/revuehistorique-2016-4-page-813.htm [lien valide en septembre 2018].

PEDERSEN Arthur, 2002, Managing tourism at World Heritage Sites. A practical manual for World Heritage Site managers, Paris, Unesco, coll. « Papers ».

Poulot Dominique, 2014, Patrimoine et Musées. L'institution de la culture, Paris, Hachette, coll. « Carré histoire ».

RICEUR Paul, 2000, La Mémoire, l'histoire et l'oubli, Paris, Éditions du Seuil, coll. « L'ordre philosophique ».

TURGEON Laurier, 2003, Patrimoines métissés. Contextes coloniaux et postcoloniaux, Paris / Québec, Éditions de la Maison des sciences de l'homme / Les Presses de l'Université Laval.

VESCHAMBre Vincent, 2007, «Patrimoine : un objet révélateur des évolutions de la géographie et de sa place dans les sciences sociales ». Annales de géographie, vol. 4, n 656, p. 361-381. Disponible en ligne, <https://www.cairn.info/revue-annales-de-geographie-2007-4-p-361.htm> [lien valide en septembre 2018].

VESCHAMBRE Vincent, 2008, Traces et mémoires urbaines. Enjeux sociaux de la patrimonialisation et de la démolition, Rennes, Presses universitaires de Rennes, coll. « Géographie sociale ».

VIDAL DE LA BLACHE Paul, 1903, Histoire de France depuis les origines jusqu'à la Révolution, vol. 1, Tableau de la géographie de la France, Paris, Hachette.

YATES Frances A., 1975 [1966], L'Art de la mémoire, Paris, Gallimard, coll. « Bibliothèque des histoires ".

\section{NOTES}

1. La totalité du texte est publié en annexe de Choay (1991).

\section{RÉSUMÉS}

Qu'apporte à la thématique générale des mémoires l'approche par la science géographique autrement dit et en première analyse, l'entrée dans ce thème par la lecture des lieux? Poser ainsi la question, c'est répondre par la singularité de cette démarche scientifique. Elle passe par la saisie, autant que faire se peut, des processus de localisation des mémoires. Elle est aussi celle des dynamiques de mémorisations : par qui et comment? Elle est enfin celle des processus d'écritures qui fait de la terre une géo-graphie, donc celle de la ou des lectures que chacun et chacune peuvent faire des mémoires dans les lieux. À son terme, cette démarche peut proposer une conclusion en cinq points qui fondent pour partie ce que peut être l'apport disciplinaire à la thématique générale. En particulier, il suggère que les notions de "monument" et de "patrimoine » ne rendent peut-être plus entièrement compte des mémoires contemporaines. Au-delà de la seule thématique mémorielle, ce parcours aboutit alors à poser quelques termes des 
processus et des dynamiques à l'œuvre dans le monde contemporain. La notion de «mémoiresMonde » en serait l'une des manifestations.

When examining the general theme of memories, what is the special usefulness of an approach by geography, in other words and more simply put, an approach by an analysis of places? To ask the question in this way is to underline the singularity of the science of geography. It is a way of trying to understand the processes by which memories are localised. It is also a way of looking at the dynamics of memorisation: memories, yes, but for whom, and how? And, finally, it is a questioning of the processes of writing that make the whole earth a geo-graphy, including the understanding - the "readings"-that each and every one of us can have of memories and places. In the end, this approach can lead to a conclusion in five points that underly, at least in part, what contribution the discipline of geography can make to the general topic. In particular, it suggests that notions of "monument" and "heritage" are no longer adequate for contemporary memories. Beyond this main issue of memories, our analysis leads us to ask further questions about the processes and dynamics in play in our contemporary world. The notion of "memoriesworld" is one manifestation of these dynamics and processes.

\section{INDEX}

Keywords : memories, monuments, heritage, memories-world, geography

Mots-clés : mémoires, monuments, patrimoine, mémoires-Monde, géographie

\section{AUTEUR}

\section{OLIVIER LAZZAROTTI}

Professeur de géographie, université de Picardie-Jules-Verne 